\title{
The rotation of Io with a liquid core
}

\author{
Jacques Henrard
}

Received: 21 October 2007 / Accepted: 20 March 2008 / Published online: 14 May 2008

(C) Springer Science+Business Media B.V. 2008

\begin{abstract}
In a previous paper (Henrard, Celest. Mech. Dyn. Astron. 178, 144-153, 2005c) we have developed an analytical theory of the rotation of the Galilean satellite Io, considered as a rigid body and based on a synthetic theory of its orbital motion due to Lainey (Théorie Dynamique des Satellites Galiléens. PhD dissertation, Observatoire de Paris, 2002) (see also Lainey et al., A\&A, 420, 1171-1183 2004a; A\&A, 427, 371-376, 2004b). One of the most important causes of departure of the actual rotation from the rigid theory is thought to be the existence of a liquid core, the size of which is unknown but would be an important piece of information concerning the structure of the interior of the satellite. In this contribution we develop the analytical theory of a liquid core contained in a cavity filled by an inviscid fluid of constant uniform density and vorticity. The theory is based on Poincaré (Bull. Astron. 27, 321-356, 1910) model and is developed by a Lie transform perturbation method, very much like in our previous contribution. Our main conclusion is that the addition of a degree of freedom (the spin of the core) with a frequency close to the orbital frequency multiplies the possibility of resonances and that for some particular size of the core one may expect a (possibly small) region of chaotic behaviour in the vicinity of the Cassini state.
\end{abstract}

Keywords Rotational dynamics · Liquid core $\cdot$ Jovian planets $\cdot$ Io

\section{Introduction}

Due to its proximity of Jupiter and the complexity of its orbits captured in the Laplacian resonance with Europe and Ganymede, the rotational motion of Io is far from an uniform rotation around a Cassini's equilibrium. We have developed in a previous contribution (Henrard 2005c) a theory based on the assumption of a rigid satellite and a model of the orbital motion given by the synthetic theory of Lainey (Lainey 2002).

J. Henrard $(\bowtie)$

University of Namur, 8, Rempart de la Vierge, 5000 Namur, Belgium

e-mail: jhenrard@math.fundp.ac.be 
The volcanic activity of Io is a clear evidence that it cannot be considered as a rigid body as the moon might be, but that the existence of a core, more or less fluid must be considered. In order to investigate the influence of such a core on the rotational motion of the satellite, we have developed a theory of the rotation of Io modeled as a rigid ellipsoidal mantle containing a cavity filled with an inviscid fluid of constant uniform density and vorticity. Poincaré (1910) provided the foundations of a conservative model of the problem. Hence our previous work can be naturally extended to include its effects. This is the aim of the present contribution.

\section{Hamiltonian formulation}

The differential equations describing the motion of a rigid mantle with a traxial ellipsoidal cavity filled with inviscid fluid of constant uniform density and vorticity, have been derived by Hough (1895), and Poincaré (1910). More recently, Touma and Wisdom (2001) have derived a Hamiltonian formulation on the base of Poincaré's formalism. We will adopt this formalism in what follows.

The components $\left(v_{1}, v_{2}, v_{3}\right)$ of the velocity field at the location $x_{i}$ inside the liquid core, in the frame of the principal axes of inertia of the mantle, are assumed to be:

$$
\begin{aligned}
& v_{1}=\left[\omega_{2}+(a / c) v_{2}\right] x_{3}-\left[\omega_{3}+(a / b) v_{3}\right] x_{2} \\
& v_{2}=\left[\omega_{3}+(b / a) v_{3}\right] x_{1}-\left[\omega_{1}+(b / c) v_{1}\right] x_{3} \\
& v_{3}=\left[\omega_{1}+(c / b) v_{1}\right] x_{2}-\left[\omega_{2}+(c / a) v_{2}\right] x_{1},
\end{aligned}
$$

where $(a \geq b \geq c)$ are the principal elliptical radii of the cavity and where $\left(\omega_{1}, \omega_{2}, \omega_{3}\right)$ are the components of the angular velocity of the mantle with respect to an inertial frame. The vector of coordinates $\left(v_{1}, v_{2}, v_{3}\right)$ specify the velocity field of the core with respect to the moving mantle. For a spherical cavity they reduce to the components of the angular velocity of the core, moving as a rigid body. Both vectors are expressed in the frame of the principal axes of inertia of the mantle. This velocity field (1) satisfy Helmholtz's equation.

The angular momentum $\vec{N}^{\prime c}$ of the core is obtained by integration of $\int(\vec{x} \times \vec{v}) \rho d W$ :

$$
\begin{aligned}
\vec{N}^{\prime c}= & \frac{M^{c}}{5}\left[\left(\frac{c}{b} v_{1}+\omega_{1}\right) b^{2}+\left(\frac{b}{c} v_{1}+\omega_{1}\right) c^{2}\right] \vec{f}_{1} \\
& +\frac{M^{c}}{5}\left[\left(\frac{c}{a} v_{2}+\omega_{2}\right) a^{2}+\left(\frac{a}{a c} v_{2}+\omega_{2}\right) c^{2}\right] \vec{f}_{2} \\
& +\frac{M^{c}}{5}\left[\left(\frac{b}{a} v_{3}+\omega_{3}\right) a^{2}+\left(\frac{a}{b} v_{3}+\omega_{3}\right) b^{2}\right] \vec{f}_{3},
\end{aligned}
$$

where $\vec{f}_{i}$ are the unit vectors along the principal moments of inertia and $M^{c}$ is the mass of the core. Considering that the moments of inertia of the core are:

$$
A^{c}=\frac{M^{c}}{5}\left(b^{2}+c^{2}\right) ; \quad B^{c}=\frac{M^{c}}{5}\left(a^{2}+c^{2}\right) ; \quad C^{c}=\frac{M^{c}}{5}\left(a^{2}+b^{2}\right),
$$

and defining the following quantities:

$$
D_{1}=\frac{2 M^{c}}{5} b c ; \quad D_{2}=\frac{2 M^{c}}{5} a c ; \quad D_{3}=\frac{2 M^{c}}{5} a b,
$$

we obtain:

$$
\vec{N}^{\prime c}=\left[A^{c} \omega_{1}+D_{1} v_{1}\right] \vec{f}_{1}+\left[B^{c} \omega_{2}+D_{2} v_{2}\right] \vec{f}_{2}+\left[C^{c} \omega_{3}+D_{3} v_{3}\right] \vec{f}_{3}
$$


The total angular momentum of the system $\vec{N}=\vec{N}^{m}+\vec{N}^{\prime c}$ is then:

$$
\vec{N}=\left[A \omega_{1}+D_{1} v_{1}\right] \vec{f}_{1}+\left[B \omega_{2}+D_{2} v_{2}\right] \vec{f}_{2}+\left[C \omega_{3}+D_{3} v_{3}\right] \vec{f}_{3},
$$

where $(A \leq B \leq C)$ are the moments of inertia of the total system.

Consider the kinetic energy of the system:

$$
\begin{aligned}
2 T= & A \omega_{1}^{2}+B \omega_{2}^{2}+C \omega_{3}^{2}+A^{c} v_{1}^{2}+B^{c} v_{2}^{2}+C^{c} v_{3}^{2} \\
& +2 D_{1} \omega_{1} v_{1}+2 D_{2} \omega_{2} v_{2}+2 D_{3} \omega_{3} v_{3},
\end{aligned}
$$

and its derivatives:

$$
\frac{\partial T}{\partial \omega_{1}}=A \omega_{1}+D_{1} v_{1} ; \quad \frac{\partial T}{\partial \omega_{2}}=B \omega_{2}+D_{2} \nu_{2} ; \quad \frac{\partial T}{\partial \omega_{3}}=C \omega_{3}+D_{3} v_{3} .
$$

We check that $\left(\partial T / \partial \omega_{i}\right)=N_{i}$, the components of the total angular momentum. On the other hand the derivatives:

$$
\frac{\partial T}{\partial \nu_{1}}=D_{1} \omega_{1}+A^{c} v_{1} ; \quad \frac{\partial T}{\partial \nu_{2}}=D_{2} \omega_{2}+B^{c} v_{2} ; \quad \frac{\partial T}{\partial \nu_{3}}=D_{3} \omega_{3}+C^{c} v_{3},
$$

are not the components of the angular momentum of the core but are close to it for a cavity close to spherical. Indeed, if we note $\left(\partial T / \partial v_{i}\right)$ by $N_{i}^{c}$, we have:

$$
N_{1}^{c}-N_{1}^{\prime c}=\left(A^{c}-D_{1}\right)\left(\omega_{1}-v_{1}\right)=\frac{M^{c}}{5}(c-b)^{2}\left(\omega_{1}-v_{1}\right) .
$$

Similar equations hold for the second and third components. The difference is thus of the second order in the departure from sphericity.

With these notations, The Poincaré-Hough's equations of motion, for the system mantlecore in the absence of external torque, are (see Touma and Wisdom 2001):

$$
\frac{d \vec{N}}{d t}=\vec{N} \times \vec{\nabla}_{\vec{N}} \mathcal{H} ; \quad \frac{d \vec{N}^{c}}{d t}=\vec{N}^{c} \times \vec{\nabla}_{-\vec{N}^{c}} \mathcal{H}
$$

The function $\mathcal{H}$ is the kinetic energy expressed in terms of the components of the vectors $\vec{N}$ and $\vec{N}^{c}$ :

$$
\begin{aligned}
\mathcal{H}= & \frac{1}{2 \alpha}\left(A^{c} N_{1}^{2}+A\left(N_{1}^{c}\right)^{2}-2 D_{1} N_{1} N_{1}^{c}\right)+\frac{1}{2 \beta}\left(B^{c} N_{2}^{2}+B\left(N_{2}^{c}\right)^{2}-2 D_{2} N_{2} N_{2}^{c}\right) \\
& +\frac{1}{2 \gamma}\left(C^{c} N_{3}^{2}+C\left(N_{3}^{c}\right)^{2}-2 D_{3} N_{3} N_{3}^{c}\right),
\end{aligned}
$$

where $\alpha=A A^{c}-D_{1}^{2}, \beta=B B^{c}-D_{2}^{2}$, and $\gamma=C C^{c}-D_{3}^{2}$.

\section{Additional approximations}

In order to define a simplified approximation, we will assume that the ellipsoid of inertia of the core and of the mantle are aligned and proportional, i.e $A^{c}=\delta A, B^{c}=\delta B$, and $C^{c}=\delta C$. Actually it may be more reallistic to assume that the core is axi-symmetric, as in (Touma and Wisdom 2001), but they assume also that the mantle is axi-symmetric which is not compatible with the spin-orbit resonance assumption. In any case we have no indication concerning the flattening of such an axi-symmetric shape and this parameter, the flattening, is probably more significant than the (small) departure from axi-symmetry. Because of the lack of knowledge of the physical parameters, our aim cannot be to provide a simulation of 
the true motion but to explore the possible effects of a liquid core. A simplified model will do that. We will also linearize the Hamiltonian (12) with respect to the "small" parameters:

$$
\epsilon_{1}=\frac{2 C-A-B}{2 C} ; \quad \epsilon_{2}=\frac{B-A}{2 C}
$$

With these notations, we have $A=C\left(1-\epsilon_{1}-\epsilon_{2}\right), B=C\left(1-\epsilon_{1}+\epsilon_{2}\right), D_{1}=A^{c}$, $D_{2}=B^{c}, D_{3}=C^{c}$, and the Hamiltonian reads:

$$
\begin{aligned}
\mathcal{H}= & \frac{1}{2 C(1-\delta)}\left[\sum_{i=1}^{3} N_{i}^{2}-2 \sum_{i=1}^{3} N_{i} N_{i}^{c}+\left(\sum_{i=1}^{3}\left(N_{i}^{c}\right)^{2}\right) / \delta\right] \\
& +\frac{\epsilon_{1}}{2 C(1-\delta)}\left[\left(N_{1}^{2}+N_{2}^{2}\right)-2\left(N_{1} N_{1}^{c}+N_{2} N_{2}^{c}\right)+\left(\left(N_{1}^{c}\right)^{2}+\left(N_{2}^{c}\right)^{2}\right) / \delta\right] \\
& +\frac{\epsilon_{2}}{2 C(1-\delta)}\left[\left(N_{1}^{2}-N_{2}^{2}\right)-2\left(N_{1} N_{1}^{c}-N_{2} N_{2}^{c}\right)+\left(\left(N_{1}^{c}\right)^{2}-\left(N_{2}^{c}\right)^{2}\right) / \delta\right] .
\end{aligned}
$$

\section{Andoyer variables}

Let us introduce the two sets of Andoyer's variables (Andoyer 1926), $(\ell, g, h, L, G, H$ ) and $\left(\ell_{c}, g_{c}, h_{c}, L_{c}, G_{c}, H_{c}\right)$. The angles $(h, K, g)$ are the Euler angles of the vector $\vec{n}_{2}$, node of the equatorial plane over the plane perpendicular to the angular momentun $\vec{N}$; the angles $(J, \ell)$ position the axis of least inertia $\left(\vec{f}_{1}\right)$ with respect to $\vec{n}_{2}$ (see Fig. 1 and Deprit 1967). Correspondingly the angles $\left(h_{c}, K_{c}, g_{c}\right)$ are the Euler angles of the vector $\vec{n}_{c}$, and $\left(J_{c}, \ell_{c}\right)$ position the axis of least inertia with respect to $\vec{n}_{c}$. The variables are $(h, g, \ell)$ and $\left(h_{c}, g_{c}, \ell_{c}\right)$ and the corresponding momenta $(H=N \cos K, G=N, L=N \cos J)$ and $\left(H_{c}=N^{c} \cos K_{c}, G_{c}=N^{c}, L_{c}=N^{c} \cos J_{c}\right)$. Expressed in Andoyer variables the components of $\vec{N}$ and $\vec{N}^{c}$ are:

$$
\begin{array}{ll}
N_{1}=\sqrt{G^{2}-L^{2}} \sin \ell, & N_{1}^{c}=\sqrt{G_{c}^{2}-L_{c}^{2}} \sin \ell_{c} \\
N_{2}=\sqrt{G^{2}-L^{2}} \cos \ell, & N_{2}^{c}=\sqrt{G_{c}^{2}-L_{c}^{2}} \cos \ell_{c} \\
N_{3}=L, & N_{3}^{c}=L_{c} .
\end{array}
$$

In order to take into account the minus sign in the differential equation for $\vec{N}^{c}$ (11), we change the sign of the $\sqrt{G_{c}^{2}-L_{c}^{2}}$ and of $L_{c}$ in (15) and obtain:

$$
\begin{gathered}
\mathcal{H}=\frac{1}{2 C(1-\delta)}\left[G^{2}+G_{c}^{2} / \delta+2 L L_{c}+2 \sqrt{\left(G^{2}-L^{2}\right)\left(G_{c}^{2}-L_{c}^{2}\right)} \cos \left(\ell-\ell_{c}\right)\right] \\
+\frac{\epsilon_{1}}{2 C(1-\delta)}\left[\left(G^{2}-L^{2}\right)+\left(G_{c}^{2}-L_{c}^{2}\right) / \delta+2 \sqrt{\left(G^{2}-L^{2}\right)\left(G_{c}^{2}-L_{c}^{2}\right)} \cos \left(\ell-\ell_{c}\right)\right] \\
-\frac{\epsilon_{2}}{2 C(1-\delta)}\left[\left(G^{2}-L^{2}\right) \cos 2 \ell+\left(G_{c}^{2}-L_{c}^{2}\right) / \delta \cos 2 \ell_{c}\right. \\
\left.+2 \sqrt{\left(G^{2}-L^{2}\right)\left(G_{c}^{2}-L_{c}^{2}\right)} \cos \left(\ell+\ell_{c}\right)\right]
\end{gathered}
$$

Let us introduce the canonical change of variables: 


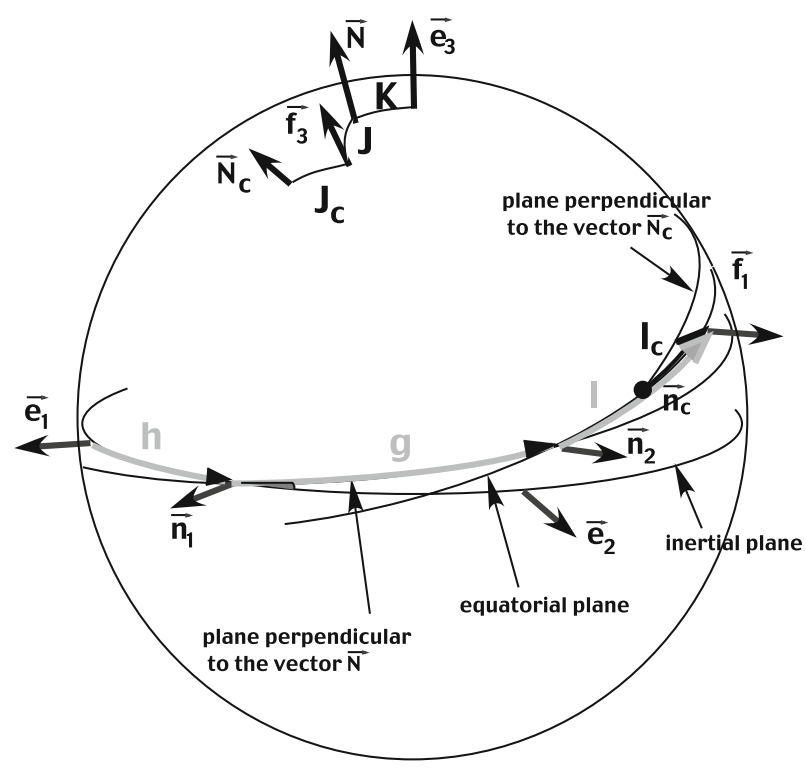

Fig. 1 The Andoyer's variables $(h, K)$ locate the plane perpendicular to the angular momentum with respect to the inertial frame $\left(\vec{e}_{1}, \vec{e}_{2}, \vec{e}_{3}\right)$ (the vector $\vec{n}_{1}$ being along the ascending node of this plane) and the angles $(g, J, \ell)$ locate the first vector of the body frame $\left(\vec{f}_{1}, \vec{f}_{2}, \vec{f}_{3}\right)$ (the vector $\vec{n}_{2}$ being along the ascending node of the equatorial plane on the plane perpendicular to the angular momentum. The variables $\left(J_{c}, \ell_{c}\right)$ locate the pseudo angular momentum of the core with respect to the mantle body frame

$$
\begin{array}{lll}
p=\ell+g+h, & P=G / N-1, & G=(1+P) N \\
q=-\ell, & Q=(G-L) / N, & L=(1+P-Q) N \\
r=-h, & R=(G-H) / N, & H=(1+P-R) N \\
p_{c}=-\ell_{c}+g_{c}+h_{c}, & P_{c}=G_{c} / N-\delta, & G_{c}=\left(\delta+P_{c}\right) N \\
q_{c}=\ell_{c}, & Q_{c}=\left(G_{c}+L_{c}\right) / N, & L_{c}=-\left(\delta+P_{c}-Q_{c}\right) N \\
r_{c}=-h_{c}, & R_{c}=\left(G_{c}-H_{c}\right) / N, & H_{c}=\left(\delta+P_{c}-R_{c}\right) N,
\end{array}
$$

of multiplier $1 / N$. The constant $N$ (resp. $\delta N$ ) is the norm $G$ (resp. $G_{c}$ ) of the angular momentum (resp. of the pseudo-angular momentum of the core). The Hamiltonian becomes:

$$
\begin{aligned}
\mathcal{H}=\frac{\omega}{2(1-\delta)}\left[P^{2}+2 P-2(1+P-Q)\left(\delta+P_{c}-Q_{c}\right)+\left(P_{c}^{2}+2 P_{c}\right) / \delta\right. \\
\left.+2 \sqrt{Q Q_{c}(2+2 P-Q)\left(2 \delta+2 P_{c}-Q_{c}\right)} \cos \left(q+q_{c}\right)\right] \\
+\frac{\omega \epsilon_{1}}{2(1-\delta)}\left[(2+2 P-Q) Q+\left(2 \delta+2 P_{c}-Q_{c}\right) Q_{c} / \delta\right. \\
\left.+2 \sqrt{Q Q_{c}(2+2 P-Q)\left(2 \delta+2 P_{c}-Q_{c}\right)} \cos \left(q+q_{c}\right)\right] \\
-\frac{\omega \epsilon_{2}}{2(1-\delta)}\left[(2+2 P-Q) Q \cos 2 q+\left(2 \delta+2 P_{c}-Q_{c}\right)\left(Q_{c} / \delta\right) \cos 2 q_{c}\right. \\
\left.+2 \sqrt{Q Q_{c}(2+2 P-Q)\left(2 \delta+2 P_{c}-Q_{c}\right)} \cos \left(q-q_{c}\right)\right] .
\end{aligned}
$$

We have used the fact that $N / C=\omega$, the norm of the angular velocity of the full body. 


\section{Librations around the equilibrium}

Let us introduce the cartesian coordinates $x=\sqrt{2 Q} \sin q, y=\sqrt{2 Q} \cos q$ and $x_{c}=$ $\sqrt{2 Q_{c}} \sin q_{c}, y_{c}=\sqrt{2 Q_{c}} \cos q_{c}$, and let us retain only the quadratic terms. We set $P=$ $P_{c}=0$, and obtain:

$$
\begin{aligned}
\mathcal{H}= & \frac{\omega}{2(1-\delta)}\left[\delta\left(x^{2}+y^{2}\right)+\left(x_{c}^{2}+y_{c}^{2}\right)+2 \sqrt{\delta}\left(y y_{c}-x x_{c}\right)\right] \\
& +\frac{\omega \epsilon_{1}}{2(1-\delta)}\left[\left(x^{2}+y^{2}\right)+\left(x_{c}^{2}+y_{c}^{2}\right)+2 \sqrt{\delta}\left(y y_{c}-x x_{c}\right)\right] \\
& +\frac{\omega \epsilon_{2}}{2(1-\delta)}\left[\left(x^{2}-y^{2}\right)+\left(x_{c}^{2}-y_{c}^{2}\right)-2 \sqrt{\delta}\left(y y_{c}+x x_{c}\right)\right] .
\end{aligned}
$$

The two degrees of freedom $(x, y)$ and $\left(x_{c}, y_{c}\right)$ are not independent of each other. In order to separate them, we use the canonical "untangling transformation" (see Henrard and Lemaître 2005):

$$
\begin{aligned}
x & =\xi_{1} \cos \beta-\xi_{2} \sin \beta, & y & =\rho\left[\eta_{1} \cos \beta+\eta_{2} \sin \beta\right] \\
x_{c} & =\rho\left[-\xi_{1} \sin \beta+\xi_{2} \cos \beta\right], & y_{c} & =\eta_{1} \sin \beta+\eta_{2} \cos \beta
\end{aligned}
$$

The multiplier of this canonical transformation is $\rho \cos 2 \beta$. Let us write the Hamiltonian (19), as:

$$
\frac{\omega}{2(1-\delta)}\left[a_{11} x^{2}+2 a_{12} x x_{c}+a_{22} x_{c}^{2}+b_{11} y^{2}+2 b_{12} y y_{c}+b_{22} y_{c}^{2}\right],
$$

with:

$$
\begin{array}{lll}
a_{11}=\delta+\epsilon_{1}+\epsilon_{2}, & a_{12}=-\sqrt{\delta}\left(1+\epsilon_{1}+\epsilon_{2}\right), & a_{22}=1+\epsilon_{1}+\epsilon_{2} \\
b_{11}=\delta+\epsilon_{1}-\epsilon_{2}, & b_{12}=\sqrt{\delta}\left(1+\epsilon_{1}-\epsilon_{2}\right), & b_{22}=1+\epsilon_{1}-\epsilon_{2} .
\end{array}
$$

After transformation, the cross product terms are:

$$
\xi_{1} \xi_{2}\left[2 a_{12} \rho-\left(a_{11}+\rho^{2} a_{22}\right) \sin 2 \beta\right]+\eta_{1} \eta_{2}\left[2 b_{12} \rho+\left(b_{11} \rho^{2}+b_{22}\right) \sin 2 \beta\right] .
$$

They disappears if $\sin 2 \beta$ is defined by:

$$
\sin 2 \beta=\frac{2 a_{12} \rho}{\left(a_{11}+a_{22} \rho^{2}\right)}=\frac{-2 b_{12} \rho}{\left(b_{22}+b_{11} \rho^{2}\right)}=\frac{-2 \sqrt{\delta}}{1+\delta}\left[1-\frac{(1-\delta)}{(1+\delta)} \epsilon_{1}\right] .
$$

The above equalities are compatible if:

$$
\rho^{2}=-\frac{a_{11} b_{12}+a_{12} b_{22}}{a_{12} b_{11}+a_{22} b_{12}}=1-2 \epsilon_{2} .
$$

After transformation the Hamiltonian becomes:

$$
\begin{aligned}
\mathcal{H}= & \frac{\omega}{2}\left\{\left[\frac{\epsilon_{1}}{1-\delta}+\frac{\epsilon_{2}}{1-\delta}\right] \xi_{1}^{2}+\left[\frac{\epsilon_{1}}{1-\delta}-\frac{\epsilon_{2}}{1-\delta}\right] \eta_{1}^{2}\right\} \\
& +\frac{\omega}{2}\left\{\left[1+\frac{\epsilon_{1}}{1-\delta}-\frac{\delta \epsilon_{2}}{1-\delta}\right] \xi_{2}^{2}+\left[1+\frac{\epsilon_{1}}{1-\delta}+\frac{\delta \epsilon_{2}}{1-\delta}\right] \eta_{2}^{2}\right\} .
\end{aligned}
$$

A change of scale $\left(\xi_{i} \rightarrow \xi_{i} \alpha_{i}\right)$ and $\left(\eta_{i} \rightarrow \eta_{i} / \alpha_{i}\right)$, with

$$
\alpha_{1}^{4}=\frac{\epsilon_{1}-\epsilon_{2}}{\epsilon_{1}+\epsilon_{2}} ; \quad \alpha_{2}^{4}=1,
$$


leads to:

$$
\mathcal{H}=\frac{\omega_{1}}{2}\left(\xi_{1}^{2}+\eta_{1}^{2}\right)+\frac{\omega_{2}}{2}\left(\xi_{2}^{2}+\eta_{2}^{2}\right)
$$

where the frequencies $\omega_{i}$ are:

$$
\frac{\omega_{1}}{\omega}=\frac{\sqrt{\epsilon_{1}^{2}-\epsilon_{2}^{2}}}{(1-\delta)} ; \quad \frac{\omega_{2}}{\omega}=1+\frac{\epsilon_{1}}{1-\delta}-\frac{\delta \epsilon_{2}}{1-\delta} .
$$

When $\epsilon_{2}=0$ (i.e. when the body and the core are axisymmetric), we recover the result of Touma and Wisdom (2001). Notice that when $\delta \rightarrow 0$, i.e. when the core vanishes, the angle $\beta \rightarrow 0$, hence the degree of freedom $\left(\xi_{1}, \eta_{1}\right)$ corresponds to the wobble of the full body and the degree of freedom $\left(\xi_{2}, \eta_{2}\right)$ corresponds to the motion of the core, which is irrelevant as the core has disappeared. In that case and when $\epsilon_{2}=0$ we have $\omega_{1} \rightarrow \epsilon_{1} \omega$ and $\omega_{2} \rightarrow\left(1-\epsilon_{1}\right) \omega$.

\section{Perturbation of Io by Jupiter}

The gravitational pull of Jupiter on the rotation of the mantle of a satellite is modelized by (see Henrard 2005c):

$$
V=C_{m} n^{2}\left[\frac{d_{0}}{d}\right]^{3}\left[1+\delta_{s}\left[\frac{d_{0}}{d}\right]^{2}\right]\left[\delta_{1}\left(x^{2}+y^{2}\right)+\delta_{2}\left(x^{2}-y^{2}\right)\right],
$$

where $d$ is the distance between the planet and the center of mass of the satellite, $d_{0}$ the mean value of this distance, $C_{m}=(1-\delta) C$ the largest moment of inertia of the mantle, $\delta_{1}=-(3 / 2)\left(n^{*} / n\right)^{2}(2 C-A-B) / 2 C$ and $\delta_{2}=-(3 / 2)\left(n^{*} / n\right)^{2}(B-A) / 2 C$. The constant $n^{*}=\sqrt{G M_{J} / d_{0}^{3}}$ is very close to the orbital frequency $n=\omega$. Dividing the potential by $\omega C$ as we did for the Hamiltonian of the free rotation, we obtain:

$$
\frac{V}{\omega C}=\omega\left[\frac{C_{m}}{C}\right]\left[\frac{d_{0}}{d}\right]^{3}\left[1+\delta_{s}\left[\frac{d_{0}}{d}\right]^{2}\right]\left[\delta_{1}\left(x^{2}+y^{2}\right)+\delta_{2}\left(x^{2}-y^{2}\right)\right] .
$$

The components $(x, y)$ of the unit vector pointing to Jupiter and the distance $d$ are computed on the basis of the synthetic theory of the motion of Io developed by Lainey (Lainey et al. 2004a,b) as in Henrard (2005c) (Tables 1, 2).

\section{Cassini states and librations}

As for most regular satellites in the Solar System, the spin period of Io is equal to its orbital period. In such a case, as Cassini pointed out, the node of the orbit and the node of the equator have, in the mean, the same period. There is thus a resonance between the angles $\lambda_{1}$ and $p$

Table 1 Constants used in the theory (Sohl et al. 2002; Zhang 2003)

\begin{tabular}{ll}
\hline$J_{2}=1.828 \times 10^{-3}$ & $C_{2}^{2}=5.537 \times 10^{-4}$ \\
$C^{\prime} / M R_{J}^{2}=0.376856$ & $R_{J} / d_{0}=0.1693$ \\
$J_{2}($ J up $)=1.475 \times 10^{-2}$ & $C^{\prime} / M_{J} R_{J}^{2}=0.26$ \\
$\delta_{1}=-7.2761 \times 10^{-3}$ & $\delta_{2}=-4.4500 \times 10^{-3}$ \\
\hline
\end{tabular}


Table 2 The angular variables in the description of Io orbit (Lainey 2002)

\begin{tabular}{llc}
\hline Angle & Frequency (in rd/day) & Phase (in rd) \\
\hline$\lambda_{1}$ & $n_{1}=3.551552283$ & 1.446157 \\
$\lambda_{2}$ & $n_{2}=1.769322710$ & 5.909624 \\
$\lambda_{3}$ & $n_{3}=0.878207924$ & 0.287366 \\
$\lambda_{4}$ & $n_{4}=0.376486233$ & 5.921262 \\
$\varpi_{1}$ & $\dot{\varpi}_{1}=2.664253678 \times 10^{-3}$ & 3.079509 \\
$\varpi_{2}$ & $\dot{\varpi}_{2}=6.779703374 \times 10^{-4}$ & 2.934505 \\
$\varpi_{3}$ & $\dot{\varpi}_{3}=1.272739669 \times 10^{-4}$ & 0.771520 \\
$\varpi_{4}$ & $\dot{\varpi}_{4}=3.206512586 \times 10^{-5}$ & 2.407105 \\
$\Omega_{1}$ & $\dot{\Omega}_{1}=-2.315095998 \times 10^{-3}$ & 2.828461 \\
$\Omega_{2}$ & $\dot{\Omega}_{2}=-5.692063559 \times 10^{-4}$ & 1.047942 \\
$\Omega_{3}$ & $\dot{\Omega}_{3}=-1.249130216 \times 10^{-4}$ & 3.326428 \\
$\Omega_{4}$ & $\dot{\Omega}_{4}=-3.056124106 \times 10^{-5}$ & 1.655000 \\
$\ell=$ Laplace lib. & $\dot{\ell}_{=3.050991204 \times 10^{-3}}$ & 0.471900 \\
$\lambda$ Sun & $n_{S}=1.450097893 \times 10^{-3}$ & 2.108700 \\
\hline
\end{tabular}

Table 3 Obliquity and frequencies (in unit of the orbital frequency) of the free librations

\begin{tabular}{llllll}
\hline & $R_{C}$ & $K^{*}($ radian $)$ & Latitude $\left(\omega_{v}\right)$ & Wobble $\left(\omega_{w}\right)$ & Core $\left(\omega_{c}\right)$ \\
\hline$\delta=0$ & 0 & $0.76198 \times 10^{-3}$ & $1.10993 \times 10^{-2}$ & $0.76969 \times 10^{-2}$ & \\
$\delta=0.1$ & 0.63 & $0.76699 \times 10^{-3}$ & $0.99242 \times 10^{-2}$ & $0.82848 \times 10^{-2}$ & 1.00691 \\
$\delta=0.2$ & 0.72 & $0.77334 \times 10^{-3}$ & $0.87491 \times 10^{-2}$ & $0.90555 \times 10^{-2}$ & 1.00930 \\
$\delta=0.3$ & 0.79 & $0.78166 \times 10^{-3}$ & $0.75740 \times 10^{-2}$ & $1.00794 \times 10^{-2}$ & 1.01219 \\
$\delta=0.4$ & 0.83 & $0.79303 \times 10^{-3}$ & $0.63989 \times 10^{-2}$ & $1.15087 \times 10^{-2}$ & 1.01594
\end{tabular}

on one hand, and the angles $\Omega_{1}$ and $r$. To take this into account,we define the two resonance angular variables $\sigma$ and $\rho$ and introduce a new set of canonical variables:

$$
\begin{array}{ll}
\sigma=p-L_{1}+\pi, & P \\
\rho=r+\Omega_{1}, & R .
\end{array}
$$

The resonant angle $\sigma$ is defined as the difference between the angle $p$ and the mean longitude of Jupiter as seen from Io. Similarly $\rho$ is defined as the difference between the angle $h=-r$ and the ascending node of the orbit of Jupiter. The variable $L_{1}$ in Lainey's theory is the mean longitude of Io as seen from Jupiter. The mean longitude of Jupiter as seen from Io is thus $L_{1}+\pi$.

The transformation being time dependent we ought to add its remainder function $-n_{1} P+$ $\dot{\Omega}_{1} R$ to the Hamiltonian.

As in (Henrard 2005a, b) we isolate $\mathcal{K}_{0}$ the part of the Hamiltonian which do not depend on the angular variables describing the perturbed motion of Io. It corresponds to a problem for which Io is on a circular Keplerian orbit around Jupiter.

Equilibria (the Cassini equilibria) are found for $\left(\sigma=\rho=x=y=x_{c}=y_{c}=0\right.$ ) and $P=P^{*}$ and $R=R^{*}$ such that the derivatives of $\mathcal{K}_{0}$ with respect to $P$ and $R$ vanish. The values of $P^{*}$ is extremely small $\left(10^{-10}\right)$ and the value of $K^{*}=\cos ^{-1}\left(1-R^{*}\right)$ for the main Cassini equilibrium is given in Table 3 for various values of $\delta$. The value of the mean radius of the core (considering that core and mantle have the same density) is also given. 
Expanding $\mathcal{K}_{0}$ in powers of the small canonical departure from the main Cassini' state:

$$
\begin{array}{ll}
\xi_{\sigma}=\sigma, & \eta_{\sigma}=P-P^{*} \\
\xi_{\rho}=\sqrt{2\left(R-R^{*}\right)} \sin r, & \eta_{\rho}=\eta_{\rho}^{*}+\sqrt{2\left(R-R^{*}\right)} \cos r \\
\xi_{w}=x, & \eta_{w}=y \\
\xi_{c}=x_{c}, & \eta_{c}=y_{c},
\end{array}
$$

where $\eta_{\rho}^{*}=\sqrt{2 R^{*}}$, and keeping only the quadratic terms, we define a linear (and thus integrable) system for which we introduce angle-action variables as in (Henrard 2005c) for the first two degrees of freedom and as in (20) for the last two:

$$
\begin{aligned}
& \xi_{\sigma}=\sqrt{2 U U^{*}} \sin u-\gamma \sqrt{2 V V^{*}} \sin v \\
& \eta_{\sigma}=(1-\alpha \gamma) \sqrt{2 U / U^{*}} \cos u-\alpha \sqrt{2 U / U^{*}} \cos u \\
& \xi_{\rho}=\alpha \sqrt{2 U U^{*}} \sin u+(1-\alpha \gamma) \sqrt{2 V V^{*}} \sin v \\
& \eta_{\rho}=\gamma \sqrt{2 U / U^{*}} \cos u+\sqrt{2 U / U^{*}} \cos u \\
& \xi_{w}=\cos \beta \sqrt{2 W W^{*}} \sin w-\sin \beta \sqrt{2 Z Z^{*}} \sin z \\
& \eta_{w}=\left[\cos \beta \sqrt{2 W / W^{*}} \cos w-\sin \beta \sqrt{2 Z / Z^{*}} \cos z\right] / \cos 2 \beta \\
& \xi_{c}=\left[-\sin \beta \sqrt{2 W W^{*}} \sin w+\cos \beta \sqrt{2 Z Z^{*}} \sin z\right] / \cos 2 \beta \\
& \eta_{c}=\sin \beta \sqrt{2 W / W^{*}} \cos w+\cos \beta \sqrt{2 Z / Z^{*}} \cos z .
\end{aligned}
$$

The parameters $\alpha, \beta, \gamma$ and the scaling factors $U^{*}, V^{*}, W^{*}, Z^{*}$ are function of $\delta$ and adjusted in such a way that, after transformation, the quadratic Hamiltonian reduces to

$$
\mathcal{N}=\omega_{u} U+\omega_{v} V+\omega_{w} W+\omega_{z} Z
$$

The frequencies of the free libration in longitude $\left(\omega_{u}\right)$, of the free libration in latitude $\left(\omega_{v}\right)$, of the wobble $\left(\omega_{w}\right)$ and of the free libration of the core $\left(\omega_{c}\right)$ are functions of $\delta$ and tabulated in Table 3. The frequency of the free libration in longitude $\left(\omega_{u}\right)$ does not depend on the value of $\delta$, and is equal to 0.133556 .

\section{Perturbation theory}

We impose on the perturbation function $\mathcal{P}=\mathcal{H}-\mathcal{N}$ the transformations described in the previous sections and expand it, up to the fourth degree, in powers of the amplitudes $\sqrt{2 U}, \sqrt{2 V}, \sqrt{2 W}, \sqrt{2 Z}$ of the oscillations around the Cassini's equilibrium. A fourth order perturbation theory by Lie transform is now straightforward except that, because of the introduction of an extra frequency $\omega_{c}$, close to the orbital frequency, several harmonics are close to be resonant. An attempt at averaging all the quasi periodic contributions is bound to fail. Instead we decided to eliminate from the Hamiltonian only the terms the frequency of which were much larger than their amplitude. This partial averaging left us with a Hamiltonian still containing quasi periodic terms such as the ones listed in Table 3. The first two are not really dangerous. The amplitudes are small and the frequencies do not go to zero. They can be eliminated in a further transformation with a rather small effect on the solution. The other terms (and some smaller ones not listed) are of the second order in the amplitudes $\sqrt{2 U}, \sqrt{2 V}, \sqrt{2 W}, \sqrt{2 Z}$, which means that the origin $(\sqrt{2 U}=\sqrt{2 V}=\sqrt{2 W}=$ $\sqrt{2 Z}=0$ ) is still an equilibrium of the partially averaged Hamiltonian. Nevertheless, the existence of those terms, the frequencies of which goes to zero for some values of the parameter $\delta$ 
Table 4 Frequencies (in unit of $10^{-2}$ ) of some resonant terms

\begin{tabular}{llrrrrr}
\hline Angular variable & Amp. & $\delta=10^{-3}$ & $\delta=0.1$ & $\delta=0.2$ & $\delta=0.3$ & $\delta=0.4$ \\
\hline$\lambda_{1}-\Omega_{1}-z$ & $1 \times 10^{-8}$ & -0.421 & -0.626 & -0.865 & -1.154 & -1.529 \\
$\lambda_{1}-\Omega_{2}-z$ & $7 \times 10^{-8}$ & -0.471 & -0.675 & -0.914 & -1.203 & -1.578 \\
$\lambda_{1}-\Omega_{1}+v-z$ & $5 \times 10^{-3}$ & 0.687 & 0.366 & 0.010 & -0.397 & -0.889 \\
$2 \lambda_{2}-\varpi_{2}-\Omega_{1}+v-z$ & $1 \times 10^{-5}$ & 0.304 & -0.016 & -0.372 & -0.779 & -1.272 \\
$\lambda_{1}-2 \lambda_{2}+\Omega_{1}-v-w$ & $1 \times 10^{-5}$ & -0.042 & 0.134 & 0.329 & 0.549 & 0.809 \\
$2 \lambda_{1}-2 \lambda_{2}+w-z$ & $3 \times 10^{-5}$ & 0.646 & 0.501 & 0.339 & 0.152 & -0.080 \\
\hline
\end{tabular}

Fig. 2 Two of the resonant frequencies: above the frequency of the angular variable $\lambda_{1}-\Omega_{1}+v-z$, below the frequency of $2 \lambda_{2}-\varpi_{3}-\Omega_{1}+v-z$

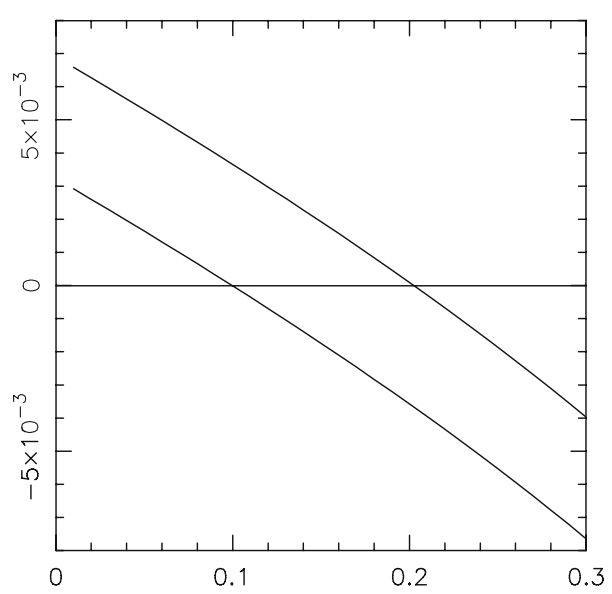

(as shown in Table 4) is an indication that the averaging process may be flawed and that the vicinity of the Cassini equilibrium may be chaotic for values of the parameter $\delta$ around $\delta=0.1$, where the frequency of the perturbing term in $\cos \left(2 \lambda_{2}-\varpi_{2}-\Omega_{1}+v-z\right)$ is close to zero, or around $\delta=0.2$, where it is the frequency of the rather large perturbing term $\cos \left(\lambda_{1}-\Omega_{1}+v-z\right)$ which vanishes, or around $\delta=0.4$, where it is the frequency of $\cos \left(2 \lambda_{1}-2 \lambda_{2}+w-z\right)$ (Fig. 2).

Even if, for particular values of the parameter $\delta$, the possibility of the existence of resonances and chaotic motions in the vicinity of the Cassini equilibrium, may cast a doubt on the validity of the perturbative approach we have taken, it may be useful to report the effects of the partial averaging. We list in Table 5 the non-linear corrections to the frequencies of the free libration (in unit of the orbital frequency). We list also in Tables 6 and 7 the expressions of $P_{1}$ and $P_{2}$, the first two component of the unit vector perpendicular to Jupiter equator (in the frame of the principal moment of inertia of Io), and, in Tables 8 and 9, the principal terms of the free librations of these two components.

Tables 6 and 7 show that in general the presence of a liquid core does not modify considerably the behaviour of the spin of Io except for some particular values of the parameter $\delta$ which are such that some combination of frequencies goes to zero implying a second order resonance. Close to these particular values (for instance for $\delta=0.2$ and $\delta=0.4$ ) new combination of angular variables appear in the Fourier expansions. 
Table 5 Non-linear corrections to the frequencies of the free librations

\begin{tabular}{lllll}
\hline & Longitude $\left(\omega_{u}\right)$ & Latitude $\left(\omega_{v}\right)$ & Wobble $\left(\omega_{w}\right)$ & Core $\left(\omega_{c}\right)$ \\
\hline$\delta=0.001$ & $-3.077 \times 10^{-8}$ & $-3.371 \times 10^{-5}$ & $-3.327 \times 10^{-5}$ & $-0.003 \times 10^{-5}$ \\
$\delta=0.1$ & $-3.083 \times 10^{-8}$ & $-3.044 \times 10^{-5}$ & $-3.045 \times 10^{-5}$ & $-0.296 \times 10^{-5}$ \\
$\delta=0.2$ & $-3.086 \times 10^{-8}$ & $-2.689 \times 10^{-5}$ & $-2.773 \times 10^{-5}$ & $-0.522 \times 10^{-5}$ \\
$\delta=0.3$ & $-3.085 \times 10^{-8}$ & $-2.340 \times 10^{-5}$ & $-2.479 \times 10^{-5}$ & $-0.675 \times 10^{-5}$ \\
$\delta=0.4$ & $-3.086 \times 10^{-8}$ & $-1.979 \times 10^{-5}$ & $-2.120 \times 10^{-5}$ & $-0.812 \times 10^{-5}$ \\
\hline
\end{tabular}

Table 6 Expressions (truncated at $10^{-2}$ ) of $10^{4} \times P_{1}$ for different values of $\delta$

\begin{tabular}{lrrrrr}
\hline & $\delta=0$ & $\delta=0.1$ & $\delta=0.2$ & $\delta=0.3$ & $\delta=0.4$ \\
\hline $\sin \left(\lambda_{1}-\Omega_{1}\right)$ & -7.620 & -7.656 & -7.478 & -7.570 & -7.702 \\
$\sin \left(\lambda_{1}-\Omega_{2}\right)$ & -1.836 & -1.815 & -1.826 & -1.850 & -1.836 \\
$\sin \left(\lambda_{1}-\Omega_{3}\right)$ & -0.344 & -0.351 & -0.335 & -0.332 & -0.331 \\
$\sin \left(\lambda_{1}-\Omega_{4}\right)$ & 0.342 & 0.349 & 0.334 & 0.331 & 0.329 \\
$\sin \left(\lambda_{1}-2 \lambda_{2}+\Omega_{2}\right)$ & -0.006 & -0.016 & -0.007 & -0.007 & -0.002 \\
$\sin \left(3 \lambda_{1}-4 \lambda_{2}+\Omega_{2}\right)$ & & -0.051 & & & -0.232 \\
$\cos \left(2 \lambda_{1}-2 \lambda_{2}+\varpi_{3}-\Omega_{2}\right)$ & & -0.021 & & -0.004 & \\
\hline
\end{tabular}

Table 7 Expressions (truncated at $10^{-2}$ ) of $10^{4} \times P_{2}$ for different values of $\delta$

\begin{tabular}{|c|c|c|c|c|c|}
\hline & $\delta=0$ & $\delta=0.1$ & $\delta=0.2$ & $\delta=0.3$ & $\delta=0.4$ \\
\hline $\cos \left(\lambda_{1}-\Omega_{1}\right)$ & -7.615 & -8.096 & -7.800 & -7.943 & -8.119 \\
\hline $\cos \left(\lambda_{1}-\Omega_{2}\right)$ & -1.835 & -1.838 & -1.843 & -1.850 & -1.859 \\
\hline $\cos \left(\lambda_{1}-\Omega_{3}\right)$ & -0.344 & -0.346 & -0.328 & -0.322 & -0.321 \\
\hline $\cos \left(\lambda_{1}-\Omega_{4}\right)$ & 0.342 & 0.344 & 0.325 & 0.320 & 0.319 \\
\hline $\cos \left(\lambda_{1}-2 \lambda_{2}+\Omega_{2}\right)$ & 0.035 & 0.017 & 0.020 & 0.013 & 0.004 \\
\hline $\sin \left(3 \lambda_{1}-4 \lambda_{2}+\Omega_{2}\right)$ & & -0.012 & & & -0.228 \\
\hline
\end{tabular}

Table 8 Free librations of $P_{1}$ (truncated at $5 \times 10^{-1}$ )

\begin{tabular}{llllll}
\hline & $\delta=0.001$ & $\delta=0.1$ & $\delta=0.2$ & $\delta=0.3$ & $\delta=0.4$ \\
\hline$\sqrt{2 V} \sin \left(\lambda_{1}-\Omega_{1}+v\right)$ & -0.994 & -0.990 & -0.990 & -0.992 & -0.992 \\
$\sqrt{2 W} \sin w$ & -0.502 & -1.371 & -0.854 & -1.009 & -1.179 \\
$\sqrt{2 Z} \sin z$ & -0.999 & -1.700 & -1.213 & -1.347 & -1.507 \\
\hline
\end{tabular}

Table 9 Free librations of $P_{2}$ (truncated at $10^{-1}$ )

\begin{tabular}{|c|c|c|c|c|c|}
\hline & $\delta=0$ & $\delta=0.1$ & $\delta=0.2$ & $\delta=0.3$ & $\delta=0.4$ \\
\hline$\sqrt{2 V} \cos \left(\lambda_{1}-\Omega_{1}+v\right)$ & -0.982 & -0.991 & -0.986 & -0.990 & -0.992 \\
\hline$\sqrt{2 W} \cos w$ & 2.008 & 1.131 & 1.610 & 1.440 & 1.281 \\
\hline$\sqrt{2 Z} \cos z$ & -0.049 & -0.331 & -0.327 & -0.349 & -0.349 \\
\hline
\end{tabular}




\section{Conclusion}

This preliminary investigation shows that the consideration of a liquid core introduces in the problem of the rotation of Io a new frequency (the frequency of the spin of the core) close to the orbital frequency. The orbital motion and the rotation of Io is already characterized by long period contributions. The introduction of a new frequency close to the main frequency multiplies the possibility of commensurability and resonance. We have detected three possible such resonances for values of $\delta$ (ratio of the core inertia to the full inertia of the body) close to $0.1,0.2$ and 0.4 . These are second order resonances which do not displace the Cassini state but might generate a chaotic vicinity around it. Of course, this is a very preliminary exploration of the effect of a liquid core on the rotation of Io. Our assumptions on the shape of the cavity containing the core may not be optimal but we do not think this should alter significantly the results. We plan to test an axi-symmetric model of the cavity in a further publication. What could be more important is our neglect of the effects of the possible dissipation occuring at core-mantle boundary. Such effects will also be investigated in further publications.

\section{References}

Andoyer, H.: Mécanique Céleste. Gauthier-Villars, Paris (1926)

Deprit, A.: Free rotation of an rigid body studied in the phase space. Am. J. Phys. 35, 424-428 (1967)

Goldreich, P., Peale, S.J.: Spin-orbit coupling in the solar system. Astron. J. 71, 425-437 (1966)

Henrard, J.: The rotation of Europa. Celest. Mech. Dyn. Astron. 91, 131-149 (2005a)

Henrard, J.: Complements to the rotation of Europa. Celest. Mech. Dyn. Astron. 93, 101-112 (2005b)

Henrard, J.: The rotation of Io. Icarus. 178, 144-153 (2005c)

Henrard, J., Lemaître, A.: The untangling transformation. Astron. J. 130(5), 2415-2417 (2005)

Hough, SS.: The oscillations of a rotating ellipsoidal shell containing fluid. Philos. Trans. R. Soc. London A 186, 469-506 (1895)

Lainey, V. Théorie Dynamique des Satellites Galiléens. PhD dissertation, Observatoire de Paris (2002)

Lainey, V., Arlot, J.E., Vienne, A.: New accurate ephemerides for the Galilean satellites of Jupiter: I Numerical integration of elaborated equation of motion. A\&A 420, 1171-1183 (2004a)

Lainey, V., Arlot, J.E., Vienne, A.: New accurate ephemerides for the Galilean satellites of Jupiter: II Fitting the observations. A\&A 427, 371-376 (2004b)

Poincaré, H.: Sur la précession des corps déformables. Bull. Astron. 27, 321-356 (1910)

Sohl, F., Spohn, T., Breuer, D., Nagel, K.: Implication from Galileo observations on the interior structure and chemistry of the Galilean satellites. Icarus 157, 104-119 (2002)

Touma, J., Wisdom, J.: Nonlinear core-mantle coupling. AJ 122, 1030-1050 (2001)

Zhang, H.: Internal structure models and dynamical parameters of the Galilean satellites. Celest. Mech. Dyn. Astron. 87, 189-195 (2003) 